\title{
Aportaciones de la Antropología al estudio de la relación hombre-medio y la producción agrícola
}

\section{Contributions of Anthropology to the study of the relation between man and the environment and agricultural production}

\author{
Gonzalo Melgar Del Corral ${ }^{1}$ \\ gonzalo.melgar@uclm.ec
}

\section{Resumen}

En el artículo se realiza un análisis histórico de las principales aportaciones de la Antropología en el estudio del problema hombre-naturaleza a partir de diversas fuentes bibliográficas. Se realiza un recorrido de la perspectiva ecológica en Antropología; el Estructuralismo y el Simbolismo, así como las aportaciones del enfoque sistémico. Posteriormente analiza la perspectiva simbólica cognitiva llegando a la Ecología Simbólica. Ya dentro de la perspectiva política recoge las concepciones del Sistema Mundial y describe las tres líneas de la Ecología Política: el Neoliberalismo, el enfoque culturalista, representado por el Ecofeminismo, y el Ecosocialismo.

Posteriormente relata las aportaciones de la Antropología al campo más concreto de la producción agrícola, hasta llegar a la Agroecología y la Etnoecología.

Finalmente, señala el interés de las recientes corrientes sociológicas y antropológicas de la emancipación social, para entender los movimientos sociales de la producción ecológica alternativa y de la recuperación de las agriculturas tradicionales.

\section{Palabras clave}

Antropología social, ecología, antropología medio ambiental, agroecología, emancipación social.

Forma sugerida de citar: Melgar Del Corral, Gonzalo (2017). Aportaciones de la Antropología al estudio de la relación hombre-medio y la producción agrícola. Universitas, $X V(26)$, pp. 87-108.

1 Docente e Investigador de la Universidad de Castilla-La Mancha, adscrito al Departamento de Enfermería, Fisioterapia y Terapia Ocupacional. orcid.org/0000-0001-8212-7168 


\begin{abstract}
In the article it is performed a historical analysis of the main contributions of Anthropology to the study of the man-nature problem using different bibliographical sources. It is carried out an overview of the ecological perspective in Anthropology; the Structuralism and Symbolism, as well as the contributions of the systemic approach. Then, the cognitive symbolic approach is analyzed reaching to the Symbolic Ecology. Inside the political approach, conceptions of the World-System are collected and the three paths of the Political Ecology are described: The Neoliberalism, the Culturalist, represented by the Ecofeminism, and the Ecosocialism.

Later, the contributions of Anthropology to the more specific field of farming production are described, until reaching the Agroecology and the Ethnoecology.

Finally, it is pointed out the interest of the recent sociological and anthropological streams of social emancipation in order to understand the social movements of alternative ecological production and the recovery of traditional farming methods.
\end{abstract}

Keywords

Social anthropology, ecology, environmental anthropology, agroecology, social emancipation.

\title{
Aportaciones de la Antropología al problema de la relación hombre-medio
}

Desde la antropología se ha mantenido tradicionalmente una visión dual cultura-medio. Evidentemente se trata del reflejo en antropología de la visión clásica occidental de la naturaleza que en el estudio y en su aplicación se ha revelado como un etnocentrismo más. Ésta ha podido hacerse evidente tras algunos estudios recientes que descubren en algunas culturas ancestrales visiones no duales.

Como algunos autores han destacado, para la antropología, el "entorno", el "medio" o "la naturaleza" ha sido un eje de vital importancia en su desarrollo y su constitución como ciencia social. Efectivamente, "la relación entre cultura y naturaleza (o entre población y entorno, si se prefiere utilizar un vocabulario ecológico-técnico) ha ocupado una parte sustancial del análisis antropológico" (Comas, 1998, p. 124). 
Si la antropología se venía ocupando de la cultura, también lo hacía de lo que se consideraba, de alguna forma "su contrapartida, la naturaleza" (Luque Baena, 1985, p. 93). Efectivamente, al menos hasta mediados del siglo XX la Naturaleza en Antropología se ha entendido como un concepto enfrentado o contrapuesto al concepto de Cultura. Se trata, por tanto, de una visión dual: cultura-naturaleza, hombre-medio, población-entorno, que en modo alguno es ajena a la concepción occidental clásica de naturaleza y medio ambiente. Esta visión para Descola incluso "impide el acercamiento realmente ecológico a la relación que existe entre los humanos y el medio ambiente" (Ingold, Pálsson, \& Mastrangelo, 2001, p. 14).

Siguiendo a Santamarina, las diferentes tradiciones antropológicas han abordado el problema de "la naturaleza" desde tres grandes perspectivas: la ecológica, la simbólica-cognitiva y la política (Santamarina Campos, 2008, p. 150). Desde estas tres perspectivas, en las últimas décadas, se han producido aportaciones relevantes para los estudios antropológicos que se centran en la problemática hombre-medio.

\section{La perspectiva ecológica}

La aportación principal de la perspectiva ecológica, en concreto de la línea de la antropología denominada "ecología cultural", ha sido la de elaborar el concepto antropológico de "entorno".

Apoyándose en la defensa de la autonomía de la cultura frente al medio de Kroeber, que la entendía como algo "superorgánico" e independiente del medio (Mintegui, 2005, p. 102), pasando por Leslie White que inicia la "perspectiva ecológica" en Antropología, Steward, al intentar conjugar los factores biológicos con los culturales sirve de bisagra entre dos modos de entender la relación entre cultura y naturaleza. Por este motivo es considerado "el padre de la Ecología Cultural". La cultura, para Steward, se explica no sólo por la propia cultura, sino por el entorno en el que esta se desarrolla (Santamarina Campos, 2008, p. 152).

Dentro de la perspectiva ecológica sus continuadores se sitúan, sin embargo, en concepciones opuestas: unos, los materialistas, considerando que el medio condiciona la cultura y otros, los idealistas, considerando que la cultura no sólo es autónoma respecto al medio, sino que lo configura. Mientras que para Harris, desde el Estructuralismo, la cultura viene a ser creada 
por las condiciones del medio convirtiéndose en "una reacción frente a los problemas prácticos de la vida terrenal" (Harris, 1982, p. 11), para Sahlins, desde el Simbolismo, la naturaleza es cultura y por lo tanto fruto de las culturas, la naturaleza es culturizada y viene a ser "una materia prima en espera de que los seres humanos le den una forma significativa y un contenido" (Santamarina Campos, 2008, p. 155).

\section{Ilustración 1 \\ Perspectiva ecológica}

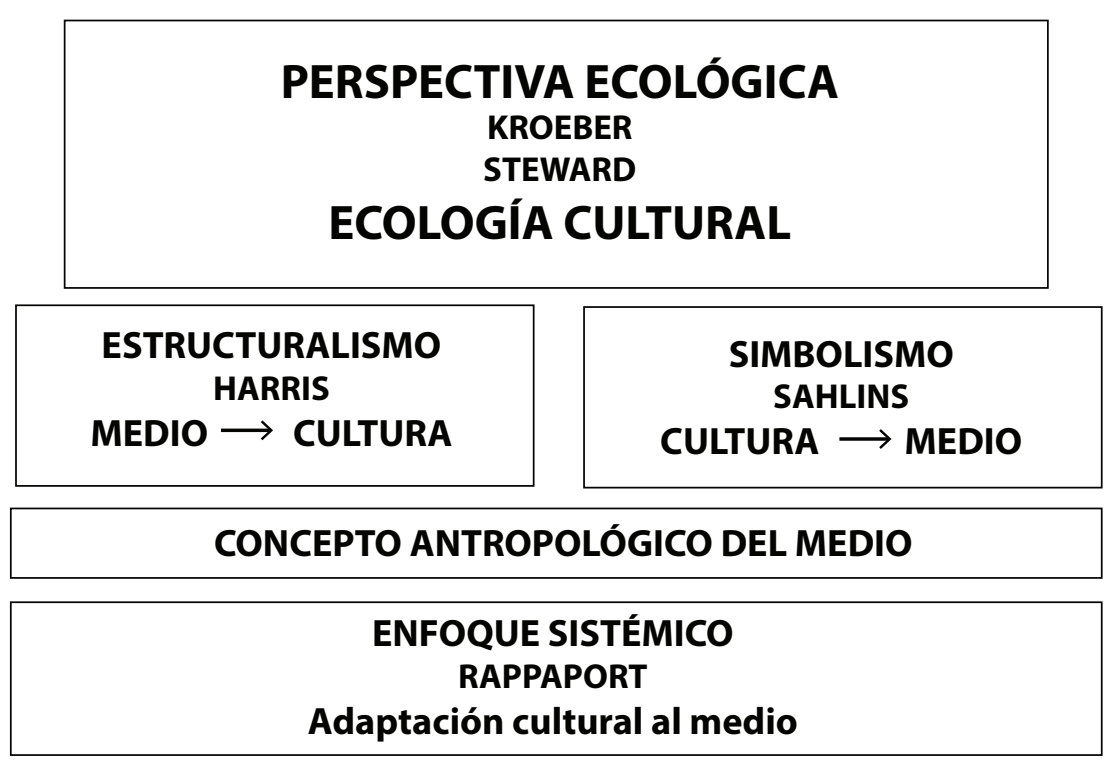

A pesar de sus diferencias tanto en el Estructuralismo como en el Simbolismo subyace una concepción dual de naturaleza-cultura (Descola \& Pálsson, 1996, pp. 2-3). Esto ha podido dificultar una visión ecológica de las relaciones entre los seres humanos y el medio ambiente, pero además ha dificultado comprender desde la Antropología los conocimientos y tecnologías de los pueblos no occidentales (Hviding, 1996, p. 169) y quizá también dificulta el estudio de los grupos que se inscriben en concepciones alternativas del binomio hombre-medio, como a nuestro juicio pueden ser los agricultores tradicionales y los alternativos. 
Un paso en la evolución de esta perspectiva ecológica en antropología se debe al enfoque sistémico de Rappaport en los años 60. Su visión de la adaptación cultural parte de contemplar al hombre como inmerso en el ecosistema, en interacción recíproca con él. Su visión es la de un sistema hombre-medio autorregulado y funcional (Rappaport \& Shapiro, 1975, p. 269), donde los seres humanos realizamos adaptaciones culturales al medio. Este concepto es similar al concepto de "adaptación al medio" de la Ecología. Algunos autores actuales han señalado que este análisis adolece del conflicto, así como de la idea de que las relaciones con el medio son una construcción social.

\section{La perspectiva simbólico cognitiva}

Otra de las perspectivas recogidas por Santamarina, es la perspectiva simbólico cognitiva. Para ésta, el individuo vive no sólo en un universo físico, sino también en un universo simbólico (Cassirer, 1945, p. 27) que le permite diferenciarse y entenderse como "otro frente al medio". Desde el estructuralismo de Levi-Strauss la naturaleza ofrecía un esquema de pensamiento para el mundo social (y así fue estudiado en el totenismo) (Santamarina Campos, 2008, p. 159) de esta forma, la dicotomía hombre-medio sirve para la interpretación antropológica.

Posteriormente, en esta misma línea, Douglas en su libro Los símbolos naturales entiende que los símbolos expresan la cosmología de una sociedad, lo que permite establecer las directrices, las normas y el control social. Douglas utiliza el término "sistema natural de simbolización" y lo define como "un sistema común a todas las culturas, recurrente y siempre inteligible" determinado por sistemas sociales afines" (Reixach, 2012, p. 182). Así, la naturaleza es culturizada. Más adelante, Victor Turner, sostiene que en la oposición hombre-medio es donde se encuentran las claves de análisis de lo social; así la naturaleza y lo social son unificados por medio del símbolo. En sus palabras, los símbolos unen lo físico con "lo estructuralmente normativo, lo orgánico con lo social” (Turner, 1980, pp. 32-33). Los modelos simbólicos de Douglas y Turner, permitieron realizar estudios simbólicos centrados sobre animales u objetos para descubrir la organización social.

Descola, introduce una nueva línea al conjugar la antropología ecológica con la antropología simbólica, bosquejando una "teoría general de ecología simbólica” (Århem, 2001, pp. 269-270). En la práctica esto rompe con la dua- 
lidad hombre medio, ya que entiende que es necesario combinar los aspectos materiales y los simbólicos, lo técnico con lo mental. En sus estudios antropológicos de la cultura Achuar, descubre que la naturaleza no es vista como "una esfera autónoma en la que los humanos sólo están presentes para producir conocimiento" (Descola, 1996, p. 93). Se trata de una visión comunitaria de lo humano, lo natural y lo sobrenatural, que aparecen sujetos a las mismas reglas. Para Descola, esto implica "una continuidad social y metonímica entre los dos órdenes” (Århem, 2001, p. 270). Esta continuidad se expresa en símbolos, rituales y prácticas. A partir de sus estudios, sostiene que la separación hombre medio, propia de occidente, "no tiene mucha importancia para otros pueblos" ya que el medio, incluyendo plantas y animales, no son vistos como objetos, sino como sujetos con "vida social". (Santamarina Campos, 2008, p. 166).

Ilustración 2

Perspectiva Simbólica

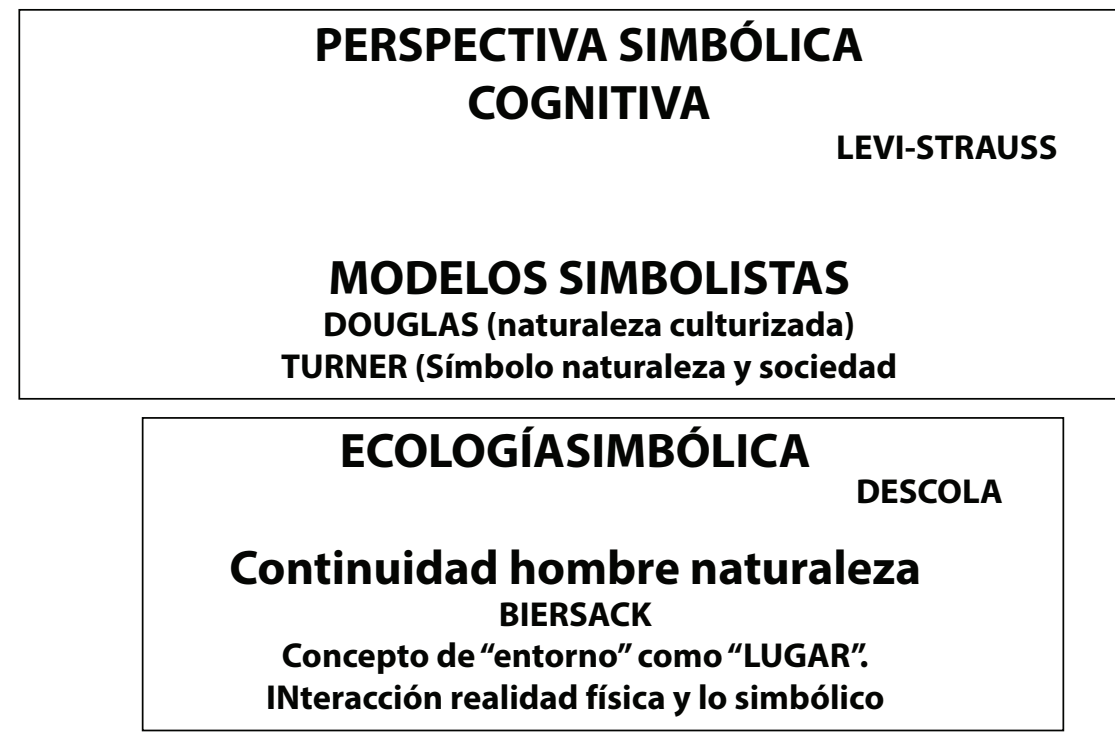

Así, la ecología simbólica de Descola ha permitido entender cómo lo simbólico y lo material interactúan. En esta línea Biersack descubre cómo la noción de "lugar" se va imponiendo en la antropología cultural y la geografía humana (Biersack, 1999, p. 81). En el "lugar" interactúa la realidad física 
y la simbólica y se constituye como una "realidad discursiva" pero también material; producto de "la imaginación humana y la historia" y por otro lado, "de la realidad material producida por las relaciones sociales", sin dejar de tener en cuenta lo global y lo intercultural (1999, p. 82).

\section{La perspectiva política}

La tercera perspectiva según señalan autores como Santamarina (Santamarina Campos, 2008, p. 169) es la denominada perspectiva política. Sin pretender ser exhaustivos, sus antecedentes se encuentran en la antropología económica, con autores como E. Wolf, con su crítica al capitalismo, el desarrollo del concepto global-local y el empleo del término ecología politica por primera vez (Biersack, 1999, p. 12); Polanyi, con sus estudios sobre mercados o Geertz con sus estudios sobre producción agrícola en relación a la colonización y la independencia (Comas, 1999, pp. 80-81).

La visión de Wolf parte de que las hegemonías ideológicas reducen los nombres a "cosas para la dominación" (Wolf \& Bárcenas, 1987, p. 20). Tenemos numerosos ejemplos de este fenómeno en la visión de los procesos agrícolas no hegemónicos (como cuando desde la agricultura industrial se califica de agricultura de subsistencia a la agricultura tradicional). Para Wolf la Historia se trasforma en "un conjunto de relaciones" que explican cómo el Capitalismo, como modo de producción, interactúa con otros modos de producción para dominarlos (1987, p. 101). Para Wolf, cada modo de producción forma una "ecología de representaciones colectivas" (Santamarina Campos, 2008, p. 170). En sus estudios "los factores sociales atraviesan todas las dimensiones del análisis y constituyen un elemento explicativo de las diferencias de acceso a los recursos en cada contexto ecológico" (Comas, 1999, p. 83). En esta línea Godelier argumenta sobre el hecho de que cada sistema económico-social determina la forma de explotación de los recursos y del trabajo humano (Godelier, 1990, p. 63). Es interesante observar cómo, al atender a los modos de producción, quedan integradas la naturaleza y la cultura, saliendo a la luz los intereses, las estrategias y el conflicto en los estudios. Otro de los autores citados como precedente de la ecología política es Geertz que identifica a los sistemas agrícolas como "ecosistemas" y analiza los factores políticos y económicos asociados a la evolución de los sistemas agrícolas, incluso de los globales (Comas, 1999, pp. 82-83). 
Esta misma autora señala que la ecología política propiamente dicha nace a raíz de la concienciación mundial de los problemas ambientales; es multidisciplinar y mantiene diferentes enfoques teóricos y describe tres paradigmas dentro de la ecología política: el neoliberal, que insiste en los límites del crecimiento, el agotamiento de los recursos y la necesidad del control demográfico; el culturalismo, representado por el ecofeminismo y, por último, el ecosocialismo, que pone el énfasis en las causas sociales y políticas de la degradación ambiental (Comas, 1999, p. 84).

El primer enfoque, el neoliberal, relaciona la elevada fecundidad con la sobreexplotación de los bienes comunitarios y ambos con la degradación ambiental. Se trata de una visión neomalthusiana y liberal que es profundamente discutida desde el enfoque ecosocialista (que veremos más adelante) y en general por la Antropología, ya que ignora que el crecimiento poblacional puede estar estimulado por el empobrecimiento, cuando la única posibilidad de las familias para incrementar la producción es incrementar la fuerza de trabajo (Comas, 1999, p. 86).

El enfoque culturalista viene representado por el ecofeminismo que Martínez Alier enmarca dentro de lo que denomina como "el ecologismo de los pobres", caracterizado por la lucha por la supervivencia en los conflictos generados por las desigualdades de acceso a los bienes ecológicos (Alier, 1994, pp. 327-328). Este ecologismo de los pobres, en consecuencia, emplea un lenguaje propio, no ecológico, que hace ver que se defiende la vida de las comunidades humanas. El ecofeminismo tienen su origen en el movimiento Chipko (desarrollado en India desde 1972) en el que se aunó la lucha popular a favor de la naturaleza con la acción de las mujeres (Comas, 1999, p. 88). El mejor exponente de esta corriente es el pensamiento de Vandana Shiva, resumido en la actualidad en el movimiento "Democracia de la Tierra" (Shiva, 2006).

El ecofeminismo entiende que "el patriarcado" es la condición de existencia del capitalismo y, por lo tanto, de la degradación ambiental y que los hombres, perdiendo la visión de que forman parte de la naturaleza, lo sustentan; así las perspectivas vitales de las mujeres les permiten sostener la lucha en defensa del medio. En este sentido, mantiene una visión dual doble (naturaleza-cultura y mujer-hombre) que ha sido criticada por determinista y esencialista (Jackson \& Pearson, 1998, p. 223), al entender la naturaleza como algo biológico, sin dimensión cultural y a la mujer como portadora de unos valores inherentes al hecho de procrear, cuidar y nutrir. Pese a estas li- 
mitaciones, el ecofeminismo facilita elementos de trasformación radical del sistema al apoyarse en la intuición, el amor, la sensibilidad como valores opuestos a la tecnología, la productividad y la competencia (Salleh, 1994, p. 47), lo que lo relaciona con la emancipación social (de la que posteriormente hablaremos).

Por otro lado, parece abocar a un supuesto "orden tradicional" no necesariamente "progresivo para la mujer" (Comas, 1999, pp. 90,91). Sin embargo, para esta autora, el Ecofeminismo aporta dos aspectos centrales que deben incluirse en cualquier análisis del sistema actual: centrarse en las aportaciones de "los grupos sin voz" y cuestionar los valores del capitalismo a partir de los mecanismos de dominación (entre ellos los que surgen de la preminencia masculina) (1999, pp. 91-92).

El ecosocialismo, según la propuesta de James O’Connor, uno de sus teóricos, aparece como una síntesis entre el marxismo y la perspectiva ecológica. Para esta corriente, el capitalismo, como sistema económico basado en la expansión constante, destruye los recursos naturales que intervienen en el proceso de producción y esta viene a ser "la contradicción básica del sistema" (O’Connor, 2001, p. 10). A diferencia de la corriente neoliberal, en el ecosocialismo se buscan explicaciones no sólo parciales sino globales a la relación entre la pobreza y la degradación ambiental (Comas, 1999, p. 92). Así por ejemplo, la estructura agraria, el acceso al capital y a la mano de obra, el mercado, la tecnología, el conocimiento de la producción, etc. afectan a las decisiones de los pequeños productores en el manejo de los recursos (Collins, 1986, pp. 138-139).

Es clave la consideración de que el medio ambiente en esta perspectiva no tiene un papel predominante, sino que se sitúa en determinadas prácticas de producción, ofreciendo posibilidades a las diferentes tecnologías y a las sociedades y soportando los efectos. Vendría por tanto a tener un papel de "posibilidad", como lo tiene en mayor medida "el sistema". Para William Durham, el impacto ambiental está mediatizado por fuerzas culturales, económicas y políticas, "dentro" y "entre" las poblaciones (Painter \& Durham, 1995). Así, el ecosocialismo en palabras de Comas, expande su análisis del uso y la distribución de los recursos naturales y las contradicciones que emergen entre sociedad y entorno (Comas, 1999, p. 95).

Como más adelante veremos, estas visiones se pueden relacionar con la Teoría del Actor Red de Latour, que al considerar como "agentes" tanto a los actores (sujetos) como a los "actantes" (elementos no humanos) sitúa al 
sujeto en un "continum" con el mundo y a la naturaleza como algo no ajeno a la actividad humana (Loredo, 2009, p. 125). Esta forma de pensar evita las contradicciones de los dualismos tradicionales (mente/cuerpo, naturaleza/ sociedad, medios/fines, etc.) (2009, p. 126).

En resumen, desde diferentes enfoques teóricos, la perspectiva de la antropología política, nos permite comprender el papel de la naturaleza en la distribución de las relaciones de poder, en un contexto marcado por la globalización y el capitalismo y como causa del deterioro del entorno.

\section{Ilustración 3 \\ Perspectiva política}

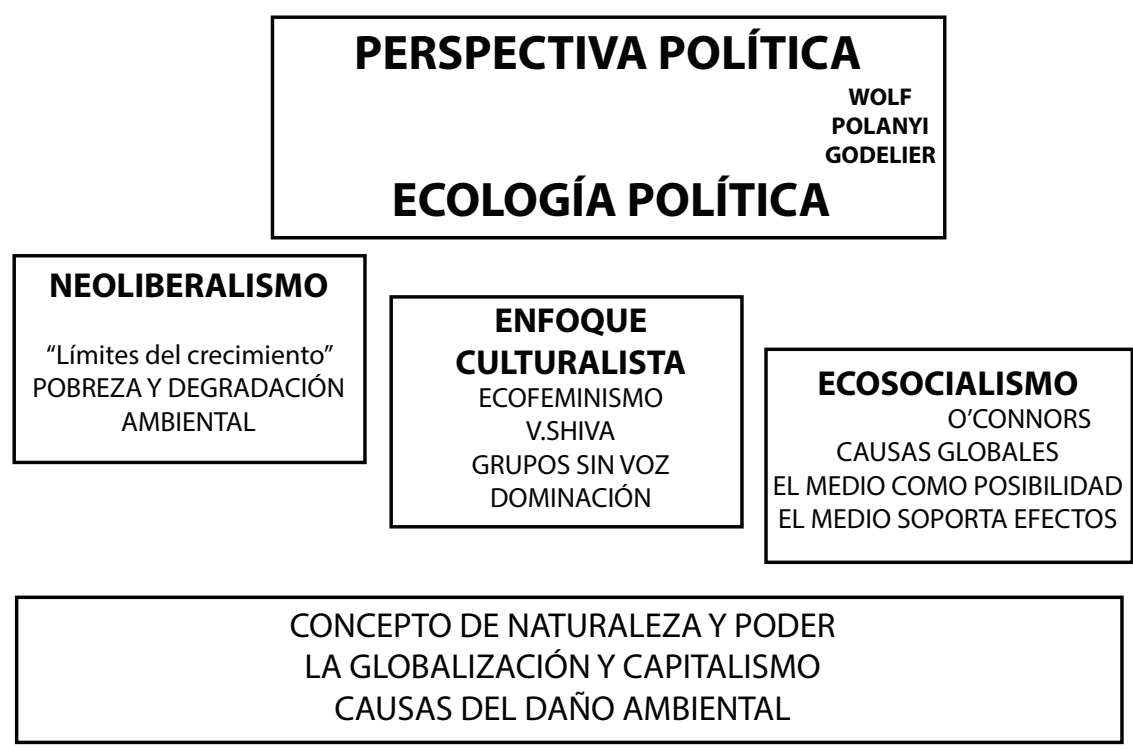

\section{Aportaciones de la Antropología: el estudio de la producción agrícola: del campesinado a la Agroecología}

Evidentemente estas concepciones sobre la relación hombre-medio han tenido su reflejo en el estudio del caso concreto de la producción de alimen- 
tos, constituyendo ésta un caso particular de estudio o un aspecto a recoger en los estudios etnográficos más generales.

En el primer caso nos encontramos con una serie de estudios antropológicos que se orientan a explicar desde el punto de vista de lo social y cultural la producción de alimentos en las diversas poblaciones. Así podemos considerar que existe toda una línea en Antropología que podríamos denominar estudios sobre el campesinado o Antropología del campesinado.

Dentro de la visión culturalista, a la que ya nos hemos referido, sobre todo en estudios de determinados ámbitos como el americano, se tendió a pasar del término "comunidades indígenas" al de "comunidades campesinas". La evolución de la antropología ha dado en superar estas visiones míticas (la de "la comunidad" y la de "lo campesino"), para concebir hoy "las comunidades como complejas redes familiares y de paisanaje que son utilizadas según estrategias que incluyen relaciones campo-ciudad" (Urrutia, 1992, p. 15).

Sin pretender ser exhaustivos, creemos que conviene repasar algunas reflexiones realizadas por la Antropología sobre los campesinos. Para hacerlo, a nuestro juicio resulta muy clarificador repasar el recorrido histórico que ya en 1980 realizaba Ángel Palerm en la primera edición de Antropología y Marxismo (Palerm, 1980). Este autor señalaba que, si bien los estudios y reflexiones sobre el campesinado fueron abundantes en Europa, la proletarización de los campesinos frente al capitalismo y también la visión histórica marxista, hizo que prácticamente desapareciera el interés por estos estudios. Sin embargo, a partir de la década de los 30 del siglo XX se reinauguran los estudios campesinos "dentro del programa boasiano de la antropología cultural norteamericana” (1980, p. 252). Es Redfield en 1930 en su monografía sobre Teploztan quien inaugura una serie amplísima de estudios sobre estas "sociedades folk". La forma de entender estas "comunidades", cercana a la visión de las "sociedades tribales", es bastante limitada ya que se concebían como sociedades tradicionales, no orientadas al cambio, con poca movilidad, no innovadoras o conservaduristas en lo cultural.

Aunque veinte años después Oscar Lewis en la misma comunidad cuestionase algunos supuestos de Redfield, es posiblemente Steward con su concepto de los "niveles de organización social" y Wolf con su "sistema mundial" quienes trasformarán la orientación de los estudios sobre el campesinado. Para Wolf el "sistema global destruye, recrea, mantiene o expande el campesinado en otras formas y en otros lugares” (Palerm, 1980, p. 271). Para el propio Palerm, sin embargo "el campesino sobrevive por medio de 
cambios adaptativos a las trasformaciones del ambiente histórico concreto" (1980, p. 271); es más, se da una articulación entre el campesinado y el capitalismo en la cual se revela el poder de supervivencia frente al sistema dominante y las adaptaciones a las condiciones del medio. Así, las formas campesinas de producción vienen a ser "reservas potenciales para la supervivencia de la especie humana” (1980, p. 278).

Palerm, destaca las aportaciones de Chayanov que señala que la unidad de análisis debe estar constituida por "el agrupamiento doméstico" y la tierra que se posee y ser vista no sólo como una unidad familiar, sino como una "organización económica". Además, la producción campesina se orienta estratégicamente no sólo al "autoabasto" sino también al "sistema capitalista", con quien se intercambian tanto las mercancías producidas, como el "trabajo excedente", a cambio de dinero. Este dinero a su vez sirve a la unidad campesina para adquirir las mercancías que ofrece el sistema capitalista (frecuentemente industriales). Con todo ello, la visión es bastante más compleja ya que la unidad campesina, de forma activa y estratégica, se modula según las necesidades del sistema, la cantidad de mercancía que produce y la cantidad de trabajo que vuelca al sistema; los bienes industriales que adquiere e incluso la cantidad y tipo de miembros que constituyen la unidad se modula para finalmente conseguir la reproducción adecuada de la unidad (1980, p. 307).

Desde esta visión compleja y articulada del campesinado, Ángel Palerm, trabajando en equipos interdisciplinares de antropólogos, agrónomos, biólogos y diversos especialistas de las ciencias naturales, se constituye en la figura clave para dar el salto integrador de la antropología con las ciencias agrarias y las ciencias naturales (Sevilla Guzmán, 1991, p. 252).

Sus seguidores, como Víctor Toledo, contribuirán a esta integración con el nacimiento de la "Agroecología" como una ciencia que aúna la Antropología, con las ciencias agronómicas y las ciencias naturales Altieri, 2002).

Siguiendo a Altieri, la Agroecología es el enfoque científico que aborda el estudio de la agricultura desde una perspectiva ecológica y se define como "un marco teórico cuyo fin es analizar los procesos agrícolas en la manera más amplia”. Se centra en el estudio de los agrosistemas entendidos como el resultado de la artificialización de la naturaleza para obtener alimentos y se entiende que "reflejan la coevolución biótica y social". Estos "etnoecosistemas se deben considerar como sistemas integrales, cuya principal característica es que otorgan legitimidad al conocimiento tradicional y experimental 
de los pueblos [...]" y dentro de él se entiende que los sistemas sociales y ambientales coevolucionan constantemente (1995, pp. 151-205).

Además, la Agroecología busca construir los fundamentos y métodos científicos de una "agricultura alternativa" (Altieri, 1995), aplicando los principios de la ciencia ecológica al diseño y manejo de agrosistemas sustentables (Gliessman, 2002). La agroecología comprende "las relaciones sociales como el elemento central de la evolución de los ecosistemas" (Sevilla Guzmán \& González de Molina Navarro, 1993, p. 9) y en particular del ecosistema agrario, entendido como un ecosistema que el hombre transforma en un "agrosistema". El agrosistema, dentro de sus límites artificialmente construidos, será eficiente, desde la perspectiva de la producción, si se mantiene en armonía con las leyes ecológicas. Precisamente de esta idea emana la crítica que la agroecología realiza de la agricultura industrial: que a largo plazo atenta contra la renovabilidad de los recursos naturales y así contra su propia base material (V. M. Toledo, Carabias, Mapes, \& Toledo, 1985).

Efectivamente para la agroecología, tomando literalmente las palabras de Víctor Toledo:

La tragedia provocada por la agricultura industrial no solo se mide por la contaminación generada por los agroquímicos que utiliza (fertilizantes, fungicidas, herbicidas, insecticidas), por la radical transformación de los hábitat originales convertidos en «pisos de fábrica» para los monótonos cultivos de una sola especie, por la dilapidación de agua, suelos y energía, por la erosión de la diversidad genética a consecuencia del uso de unas cuantas variedades mejoradas, por el incremento del riesgo a causa de los organismos transgénicos, o por la generación de alimentos peligrosos e insanos; también se distingue por un impacto cultural de incalculables consecuencias: la destrucción de la memoria tradicional representada por los saberes acumulados durante por lo menos 10.000 años de interacción entre la sociedad humana y la naturaleza (V. Toledo, 2005).

Por lo tanto, la tragedia tiene una dimensión cultural, por eso para la agroecología es de sumo interés la antropología ecológica, (junto con la etnobiología, la geografía ambiental o la agronomía), para comprender las formas no occidentales de producir alimentos, esto es, las formas alternativas de "apropiación de la naturaleza".

Toledo propone la "etnoecología" para estudiar "desde su enfoque holístico y multidisciplinario, "el complejo integrado por el conjunto de creencias 
(cosmos), el sistema de conocimientos (corpus) y el conjunto de prácticas productivas (praxis)"(V. Toledo, 2005). Así, la etnoecología pretende incorporar de forma consciente y sistemática la perspectiva cultural y antropológica en los estudios. En este sentido resulta relevante el estudio de la construcción de los espacios en relación a los sistemas de producción, actividades, tecnologías, información, valores e ideas que se plasman en "los paisajes".

Sintetizando podemos decir que la agroecología se apoya en cinco supuestos principales:

- La crítica a la agricultura industrial.

- La idea de que la conservación y reproducción de los sistemas agrarios depende de la relación existente entre los tipos de sociedades y las relaciones entre los grupos sociales de cada sociedad.

- El interés por el análisis de las desigualdades sociales como enfermedades ecosistémicas de la sociedad.

- La forma de ver las cultural tradicionales como sistemas eficientes de manejo de los recursos naturales.

- La idea de que la cultura campesina en relación a la naturaleza, tiene un aspecto de "Economía Moral" que involucra a la concepción hombre medio.

Ahondando en el asunto, para Toledo la agricultura industrializada surge de la revolución industrial, desde la ortodoxia de la ciencia moderna. La agricultura industrializada se impone en la mayoría del mundo pasando por encima de los conocimientos locales, que son visualizados como atrasados, arcaicos, primitivos o inútiles. Así, fruto de la modernidad la agricultura industrializada arrasa literalmente la memoria de la especie humana en cuanto a sus relaciones históricas con la naturaleza, revelando uno de sus rasgos principales: "su desdén, e incluso su irritación, por todo aquello considerado como tradicional" (V. Toledo, 2005, p. 1). Resistirse a la agricultura industrializada es resistir al "progreso", el "desarrollo" o la "modernización", que se funda en el mito de la supuesta superioridad de lo "moderno", del mercado y de la tecnología y la ciencia contemporáneas, sobre todo lo "tradicional".

Una consecuencia es que, a partir de estos enfoques, la relación entre los movimientos del campesinado y los procesos emancipatorios se hace obvia.

Ya en 1992 Víctor Toledo analizaba la vinculación de los movimientos campesinos de tipo ecológico (sobre todo indígenas mexicanos) con la Uto- 
pía y la emancipación (Toledo, 1992). Para Toledo, en el marco de la crisis ecológica global, y el fracaso de los sistemas de producción moderna, el campesinado tradicional está destinado a ser protagonista, ya que podría amortiguarla o incluso resolverla (1992, p. 2). Toledo advierte que es posible ya que se dan algunos fenómenos: las reflexiones técnicas de inspiración ecológica; el papel de ONGD alternativas y ecológicas; la aparición de nuevos canales de circulación de productos hacia "consumidores verdes" y la creciente influencia de los movimientos ambientalistas urbanos en el ámbito rural.

\section{Aportaciones de la Antropología a los movimientos ecológicos de producción: emancipación social y producción agrícola}

A partir de relación que acabamos de ver entre campesinado y emancipación social, resulta interesante sintetizar las aportaciones de la antropología y la sociología en la comprensión de los movimientos utópicos y emancipatorios en general para, posteriormente aplicarlo a los movimientos ecológicos en la producción agrícola.

La aproximación utópica, estudia las configuraciones culturales como "anticipaciones fragmentarias de una forma de convivencia mejor" (Krotz \& Cabrera Luna, 2002, p. 405). El trabajo de Krotz, sobre la utopía y las experiencias utópicas resulta coincidente en muchos aspectos con los postulados y la línea de investigación del sociólogo Boaventura de Sousa Santos referida a la emancipación. Santos entiende que la emancipación ha de argumentar contra la ciencia y el derecho, de manera que "tiene que reclamar una utopía" (2003, pp. 378-379).

En todo esto cobra especial importancia el concepto de hegemonía, que no se basa necesariamente en el convencimiento sino más bien, en palabras de Gimeno (Gimeno Martín, 2012, p. 146), en que la gente acepte "la desigual distribución de poder, riqueza o libertad". Los mecanismos empleados, siguiendo a este autor, son tanto el miedo como la eliminación de plantear alternativas, aparecer como la única opción de organización posible. De estos mecanismos derivaría el "consentimiento" al orden del sistema.

Krotz sintetiza las características de los actores de las experiencias utópicas del siguiente modo: los actores reconocen la coacción del sistema y se rebelan o reaccionan contra ella; proponen y practican, contra la hegemonía de la ciencia occidental, formas de conocimiento alternativas; sustancial- 
mente son culturalmente diversos o alternativos; viven su experiencia como "total", es decir, no sólo circunscrita a un ámbito; y están proponiendo una nueva identidad, contra la opción realista (Krotz, 1988, p. 35). Para Guattari los actores de experiencias emancipadoras parecen poseer cierta "capacidad de resistencia" hacia lo que él denomina "el laminado capitalístico de las subjetividades" (Richard, 2008). Serían sujetos que individual y colectivamente realizan actos de autonegación del ser impuesto por el orden o el sistema y construyen la autodeterminación personal y común (Gimeno Martín, 2012, p. 150).

Siguiendo a Gutiérrez Aguilar, los procesos emancipatorios se ven como acciones sociales auto-determinativas, personales y colectivas. Los actores desde el compromiso con la no-opresión, con la no anulación de uno mismo y del otro, se esfuerzan por no transigir, por subvertir el orden impuesto, por resistir en comunidad (Gutiérrez Aguilar, 2006).

Para Escobar, (aunque referido a experiencias decolonizadoras afrocolombianas), estas experiencias parecen enraizarse en visiones de la lucha y del futuro locales, "basadas en el lugar" y las relaciones entre el lugar y el mundo (Escobar, 2010). Para él, estos actores aparecen como "activistas" vinculados con el compromiso continuo con la realidad diaria del lugar y sus identidades surgen de una experimentación implicada (Escobar, 2010). Esto hace que estudiar sus identidades permita al antropólogo comprender y explicar la historia y el mundo a partir del lugar concreto de sus experiencias (Gimeno Martín, 2012) y buscar el "pensamiento de frontera" que en ellas se da. El primer paso que supone una cierta ruptura metodológica, según Gimeno, supone reconocer que existe una pluralidad de conocimientos, negados por la política y la ciencia, que se dan en los espacios subalternos y que se expresan con lenguajes fronterizos (Gimeno Martín, 2012, p. 164).

En este contexto tiene especial relevancia el Proyecto denominado "Modernidad/Colonialidad/Decolonialidad" (Proyecto "MCD”). Este proyecto agrupa a intelectuales latinoamericanos de distintas disciplinas y tiene por objeto "el análisis crítico de la construcción del mundo moderno" (Gimeno Martín, 2012, p. 146); para ello identifican el proceso de coacción y hegemonía ejercido por la modernidad con la colonialidad y la respuesta emancipadora con la decolonialidad. El movimiento se sitúa equidistante entre los dos enfoques clásicos a los que ya nos hemos referido: la perspectiva marxista del "Sistema Mundo" y los estudios poscoloniales de enfoque cultu- 
ral que dan más importancia al análisis de los discursos, la subjetividad y lo simbólico (Gimeno Martín, 2012, p. 154).

A partir de todo esto, podemos aplicar las reflexiones teóricas sobre la emancipación social al estudio antropológico de los movimientos de la agricultura ecológica incluida la recuperación de la tradicional y especialmente a los grupos de agricultores alternativos.

Los movimientos "ecologistas" o "ambientalistas" en su "diversidad de teorías y prácticas" se constituyen como "una nueva forma de movimiento descentralizado, multiforme, articulado en red y omnipresente" (Castells, 1998, p. 2). Así, los movimientos de la agricultura ecológica alternativa pueden entenderse como un movimiento "contracultural", en el sentido en que este autor lo entiende, ya que practican un "intento deliberado de vivir de acuerdo con normas diferentes y hasta cierto punto contradictorias de las aplicadas institucionalmente por la sociedad y de oponerse a esas instituciones basándose en principios y creencias alternativos" (Castells, 1998, p. 5)33.

Los actores de estos movimientos ecológicos de producción, practican una agricultura y ganadería alternativas, aunque en ocasiones, como ocurre en el caso europeo de forma muy mayoritaria, carezcan de referentes culturales ancestrales propios como para practicar una agricultura tradicional. Así en muchos casos toman como base un corpus de conocimientos híbridos, complementarios, alternativos y generalmente opuestos a los de la ciencia agronómica y veterinaria moderna. Desde la antropología podemos entenderlos como "activistas basados en el lugar" que redescubren o ensayan cosmovisiones que sostienen esas prácticas.

Aplicando las aportaciones de la antropología en la emancipación social, estos actores se entenderían como protagonistas de experiencias utópicas, que resisten los procesos de coacción del subsistema, especialmente el

2 Castell emplea el término "medioambientalismo" haciendo referencia a "todas las formas de conducta colectiva que, en su discurso y práctica, aspiran a corregir las formas de relación destructivas entre la acción humana y su entorno natural, en oposición a la lógica estructural e institucional dominantes", teniendo, por tanto, un componente más práctico. Mientras que se refiere con el término "ecología", a la serie de creencias, teorías y proyectos que consideran a la humanidad un componente de un ecosistema más amplio y desean mantener el equilibrio del sistema en una perspectiva dinámica y evolucionista, por lo que tiene un componente más teórico (Castells, 1998, p. 2)

3 Algunos autores proponen como más adecuada la traducción "cultura a la contra" ya que los practicantes de la contracultura no aspiran a ser una manifestación dedicada a ir en contra de la cultura, "sino más bien su intención es ser una manifestación cultural específica que camina en sentido opuesto a la cultura tradicional y oficial" (Tobar et al., 2001, p. 24). 
de la agricultura industrializada, de mercado, hegemónica, de la que intentan emanciparse.

En ocasiones puede costar descubrir a estos actores como formando parte de un fenómeno cultural o un movimiento ya que la heterogeneidad que exhiben es notable, pero ciertos criterios les definen como unidad frente al resto: sobre todo lo ecológico en la producción llevado hasta el purismo que obliga a situarse frente al "sistema" o en sus márgenes (Gómez-Ullate García de León \& Sanmartín Arce, 2004, p. 29).

Comprender estas experiencias de producción ecológica alternativa desde una aproximación utópica; entenderlas como configuraciones culturales fragmentarias pero anticipatorias de una forma de convivencia hombre-medio más sostenible saludable y mejor, puede ser la clave para contribuir desde las ciencias sociales, en especial la antropología, a darles la visibilidad que el sistema les niega.

\section{Conclusiones}

Para acercarse desde las ciencias sociales al problema hombre-medio puede ser útil recorrer la tradición antropológica: desde las aportaciones de la ecología cultural, que pretendía establecer las relaciones entre cultura y medioambiente; la consideración de que los sistemas culturales "funcionan a través de flujos de materia, energía e información"; la superación del determinismo ambiental; los esquemas de los funcionalistas ecológicos (derivados de la teoría de sistemas) y el papel que tiene la toma de decisiones en relación al ambiente.

Hoy en día, se hará necesario recurrir al marco conceptual que ofrece la Agroecología y la Etnoecología, como un puente entre la Ecología y el estudio de las prácticas agrícolas.

Además, especialmente en contextos que pretenden la trasformación de los ecosistemas, como los que proponen los movimientos ecológicos en la producción, será imprescindible contemplar las reflexiones de la Ecología Política y analizar el papel del poder en la relación entre la cultura y el ecosistema y atender a la toma de decisiones y a las actividades individuales de los actores sociales. Será imprescindible entender que estos actores se encuentran en oposición y en conflicto con las concepciones de un Sistema Mundial, que basado en el sistema económico trata de imponerse sobre las 
formas culturales más marginales (como la agricultura tradicional o la producción ecológica alternativa).

Para estos contextos marginales de producción, las reflexiones de la emancipación social permiten el reconocimiento como significativas de las experiencias alternativas campesinas (prácticas tradicionales, agricultura ecológica alternativa), que se dan cada vez con más frecuencia en el ámbito de la producción agrícola como alternativa a la producción agrícola industrial. Además, esto permitirá estudiarlas como parte de un fenómeno social global de suma importancia para la humanidad y el medio, para la relación entre hombre y naturaleza.

\section{Bibliografía}

Alier, J. M. (1994). De la economía ecológica al ecologismo popular (Vol. 60). Icaria Editorial.

Altieri, M. A. (1995). El "estado del arte" de la agroecología y su contribución al desarrollo rural en América Latina. En: Alfredo Cadenas (Ed.), Agricultura y desarrollo sostenible (pp. 151-203). Madrid: Ministerio de Agricultura, Pesca y Alimentación.

(2002). Agroecology: the science of natural resource management for poor farmers in marginal environments. Agriculture, ecosystems \& environment, 93(1), 1-24.

Århem, K. (2001). Ecocosmología y chamanismo en el Amazonas: variaciones sobre un tema. Revista Colombiana de Antropología, 37, 268-288.

Biersack, A. (1999). The Mount Kare python and his gold: Totemism and ecology in the Papua New Guinea Highlands. American Anthropologist, 101(1), 68-87.

Cassirer, E. (1945). Antropología filosófica. FCE.

Castells, M. (1998). El reverdecimiento del yo: el movimiento ecologista. La factoría (5), 135-158.

Collins, J. L. (1986). Asentamiento de pequeños propietarios de Sud-américa tropical: las causas sociales de la destrucción ecológica. Estrategias productivas y recursos naturales en la Amazonía, 138-168. Lima, CIPA.

Comas d'Argemir (1998). Antropología económica. Barcelona: Ariel.

(1999). Ecología política y antropología social. Áreas: Revista de Ciencias Sociales (19), 79-100. 
Descola, P. (1996). In the society of nature: a native ecology in Amazonia (Vol. 93). Cambridge University Press.

Descola, P., \& Pálsson, G. (1996). Nature and society: anthropological perspectives. Routledge.

Escobar, A. (2010). Territorios de diferencia. Lugar, movimientos, vida, redes. Popayán: Envión.

Gimeno Martín, J. C. (2012). Reflexiones críticas, desde los márgenes, sobre la producción de conocimientos para una acción transformadora. CulturaHombre-Sociedad CUHSO, 22(2), 141-180.

Gliessman, S. R. (2002). Agroecología. Procesos ecológicos en agricultura sostenible. CATIE. Centro Agronómico Tropical de Investigación y Enseñanza. Costa Rica.

Godelier, M. (1990). Lo ideal y lo material. Revista de Occidente (106), 5-14.

Gutiérrez Aguilar, R. (2006). A desordenar!: por una historia abierta de la lucha social. Casa Juan Pablos.

Gómez-Ullate García de León, M., \& Sanmartín Arce, R. (2004). Contracultura y asentamientos alternativos en la España de los 90 un estudio de antropología social. Retrieved from http://dialnet.unirioja.es/servlet/ exttes?codigo $=17181$

Harris, M. (1982). El materialismo cultural. Alianza Editorial.

Hviding, E. (1996). Nature, culture, magic, science. Nature and society. Anthropological perspectives, 165-184.

Ingold, T., Pálsson, G., \& Mastrangelo, S. (2001). Naturaleza y sociedad: perspectivas antropológicas: Siglo XXI.

Jackson, C., \& Pearson, R. (1998). Feminist visions of development: gender analysis and policy. Routledge.

Krotz, E. (1988). Utopía (2. ed.). México, D.F.: Universidad Autónoma Metropolitana.

Krotz, S., \& Cabrera Luna, C. L. (2002). La otredad cultural entre utopía y ciencia: un estudio sobre el origen, el desarrollo y la reorientación de la antropología. México: Fondo de Cultura Económica [etc.].

Loredo, J. C. (2009). ¿Sujetos o “actantes”? El constructivismo de Latour y la psicología constructivista. AIBR-Revista de Antropologia Iberoamericana, 4(1), 113.

Luque Baena, E. (1985). Del conocimiento antropológico: Centro de Investigaciones Sociológicas (CIS).

Mintegui, M.C.D. (2005). Procesos culturales: una aproximación desde la antropoligía social y cultural. Norba. Revista de historia(18), 93-116. 
O’Connor, J. R. (2001). Causas naturales: ensayos de marxismo ecológico: Siglo XXI.

Painter, M., \& Durham, W. H. (1995). The Social Causes of Environmental Destruction in Latin American: University of Michigan Press.

Palerm, Á. (1980). Antropología y marxismo. México: Nueva Imagen.

Rappaport, R., \& Shapiro, H. (1975). Naturaleza, cultura y antropología ecológica. Hombre, cultura y sociedad.

Reixach, J. F. (2012). Cosmologías, patrimonialización y eco-símbolos en el Pirineo catalán en un contexto global. Revista de Antropología Social, 21, 173-196.

Richard, N. (2008). Debates críticos en América Latina: 36 números de la" Revista de crítica cultural"(1990-2008) (Vol. 3): Editorial ARCIS.

Salleh, A. (1994). Naturaleza, mujer, trabajo, capital: la más profunda contradicción. Ecología política, 35-47.

Santamarina Campos, B. (2008). Antropología y medio ambiente. Revisión de una tradición y nuevas perspectivas de análisis en la problemática ecológica. AIBR: Revista de Antropología Iberoamericana, 3(2), 144-184.

Santos, B. d. S. (2003). Crítica de la razón indolente: contra el desperdicio de la experiencia : para un nuevo sentido común: la ciencia, el derecho y la política en la transición paradigmática. Bilbao: Desclée de Brouwer.

Sevilla Guzmán, E. (1991). Una propuesta de desarrollo rural endógeno para Andalucía. Revista de estudios regionales (31), 251-264.

Sevilla Guzmán, E., \& González de Molina Navarro, M. L. (1993). Ecología, campesinado e historia: La piqueta.

Shiva, V. (2006). Manifiesto para una democracia de la tierra: justicia, sostenibilidad y paz.

Tobar, L., Gallardo, R., Hadjiconstantis, M., Horno, A., Pérez, P., \& Da Silva, J. (2001). Taller de estrategias de investigación: Universidad ARCIS, Centro de Investigaciones Sociales.

Toledo, V. M (2005). La memoria tradicional: la importancia agroecológica de los saberes locales. LEISA Revista de Agroecología, 20(4), 16-19.

(1992). Utopía y naturaleza. El nuevo movimiento ecológico de los campesinos e indígenas de América Latina. Nueva Sociedad, 122, 72-85.

Toledo, V. M., Carabias, J., Mapes, C., \& Toledo, C. (1985). Ecología y autosuficiencia alimentaria. México: Siglo Veintiuno Editories 118 p. ISBN 968231349X Sp List of, 70.

Turner, V. W. (1980). La selva de los símbolos: aspectos del ritual ndembu. Siglo XXI de España Editores. 
Urrutia, J. (1992). Comunidades campesinas y antropología: historia de un amor (casi) eterno. Debate agrario, 14, 1-16.

Wolf, E. R., \& Bárcenas, A. (1987). Europa y la gente sin historia. México: Fondo de Cultura Económica.

Fecha de recepción: 5/08/2016; fecha de aceptación: 29/11/2016;

fecha de publicación: 30/06/2017 